The Be Phenomenon in Early-Type Stars, IAU Colloquium 175

ASP Conference Series, Vol. 214, 2000

M. A. Smith, H. F. Henrichs, and J. Fabregat, eds.

\title{
Can Magnetic Fields Be Detected in Be Stars?
}

\author{
Gautier Mathys \\ European Southern Observatory, Casilla 19001, Santiago 19, Chile
}

Myron A. Smith

STScI/CSC, Space Telescope Science Institute, 3700 San Martin Drive, Baltimore, MD 21218, USA

\begin{abstract}
The results of observations aimed at detecting magnetic fields in the Be star $\lambda$ Eri are reported. The observational data are analyzed both through application of an approximate analytical method and through computation of a number of simple numerical models in view of deriving constraints on the magnetic fields. General conclusions are drawn about future prospects for magnetic field detections in Be stars.
\end{abstract}

\section{Introduction}

Interest for the possible presence of magnetic fields in Be stars has existed for a long time. In his pioneering systematic search for magnetic fields in stars of various types, Babcock (1958) had observed a dozen Oe/Be stars. He had found no more than marginal evidence for the presence of magnetic fields in only two of them; these detections have never been confirmed since. Until recently, the most accurate and extensive investigation of the presence of magnetic fields in $\mathrm{Be}$ stars has been the work of Barker et al. (1985). These authors failed to achieve any detection of non-zero mean longitudinal magnetic fields in the 15 studied stars, with measurement standard deviations of the order of $100 \mathrm{G}$. This result gives an indication that large-scale organized magnetic fields with strengths in excess of few hundred gauss are not widespread among Be stars.

Any further attempt to detect fields in those stars must therefore aim either at achieving a considerably better accuracy or at applying methods that are sensitive to smaller-scale field structure. Indeed, the mean longitudinal magnetic field (as derived in the above-mentioned studies of Babcock and of Barker et al.) is the line-intensity weighted average over the visible stellar disk of the component of the magnetic vector along the line of sight. If the magnetic field has a complex structure over the stellar surface, the contributions to the observable average of regions where the field points in opposite directions will tend to cancel out mutually. Therefore, a low upper limit on the longitudinal field may correspond either to a situation where indeed no strong magnetic fields are present on the star, or to one where strong fields exist locally, but with a topology such that their net effect vanishes in disk-integrated observations. 


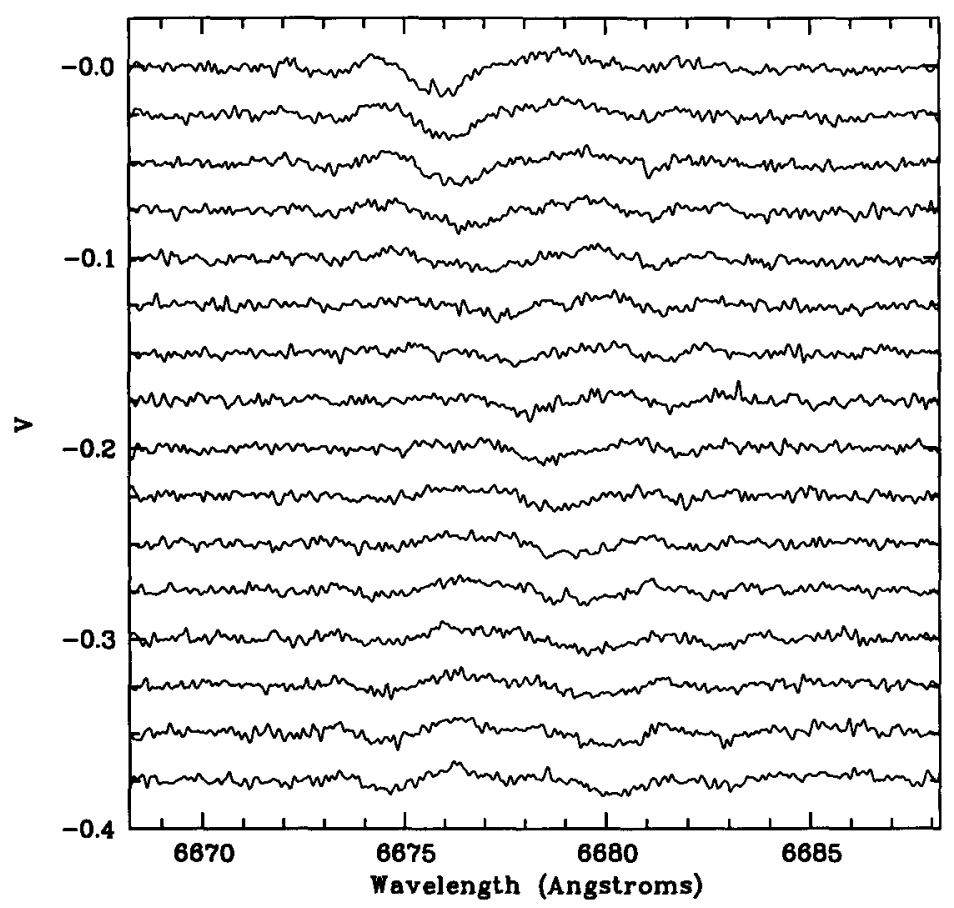

Figure 1. Time-series of Stokes $I$ observations obtained over the night of January 19-20, 1997, showing the difference between individual spectra and the average of all the Stokes $I$ spectra recorded during that night. The 16 spectra shown here have been obtained consecutively, from top to bottom; the exposure time for each exposure was 4 minute, and the time between the middle of two consecutive exposures was of the order of 6 minutes. For clarity, each tracing has been shifted in ordinate by -0.02 with respect to the previous one.

The approach which consists in achieving better accuracy has been followed in the study of $\beta$ Cep reported by Henrichs (these proc.), taking advantage of the exceptionally (for a Be star) sharp profiles of its spectral lines. Because it relies on a favourable circumstance which is not met in the vast majority of Be stars, its applicability to other cases is quite restricted, at least in the current state of instrumental developments. In this paper, we report an attempt to apply the above-mentioned alternative approach towards detecting localized magnetic features on the surface of the prototypical Be star $\lambda$ Eri. In the next section, we describe the observational strategy and the underlying assumptions. In Sect. 3, an analysis of the obtained data based on analytical considerations is presented, and in Sect. 4, constraints are derived from the comparison of the observations with simple numerical simulations. The conclusions reached and the resulting prospects for future progress are discussed in Sect. 5. 


\section{Spectropolarimetric observations of $\lambda$ Eri}

Several lines of evidence suggest that variability in the mild B2e star $\lambda$ Eri may have its origin in magnetic dissipation processes. This evidence includes an Xray flare (Smith et al. 1993) and rapid variability of absorption features known as "dimples" and, less frequently, weak emissions in its $\lambda 6678$ and other He I lines. Emissions can be produced in photospheric regions heated to $50-75 \mathrm{kK}$ (Smith et al. 1994). Similarly, Smith \& Polidan (1993) have produced models for dimples that require the presence of elevated, optically thick slabs (in the line) having an area of $\sim 4 \%$ of the stellar disk. Violent magnetic dissipative processes appear as the most likely origin of either of these phenomena. In that scenario, dimples are produced from an ablation of photospheric material caused by a nearby flare. The evaporating material is released from the surface only to be trapped and cool in an overlying magnetic loop. The material flows back to the star along the loop flux lines and the dimple feature dissipates. Magnetic pressure balance arguments together with a determination of the dimple-slab masses $\left(6 \times 10^{-13} \mathrm{M}_{\odot} \mathrm{yr}^{-1}\right)$ lead to an estimated field strength of $2 \mathrm{kG}$ at the postulated loop footpoints (Smith et al. 1996).

Accordingly, $\lambda$ Eri appeared as a prime candidate for the detection of localized, transient magnetic fields, by searching possible correlations between circular polarization and spectral transients, where and when the latter occur in the line He I $\lambda 6678$.

Observations to that effect were obtained with the ESO $3.6 \mathrm{~m}$ telescope, the Cassegrain Echelle Spectrograph CASPEC, and its built-in Zeeman analyzer (this is the post-1995 configuration described by Mathys \& Hubrig 1997). Three half nights were devoted to that project in January and February 1997. During each night, time series of spectra were recorded simultaneously in right and left circular polarizations. Adverse meteorological conditions limited the number of spectra we could obtain on each night: 11,16 , and 6 , respectively, with exposure times between 4 and 10 minutes. The achieved signal-to-noise ratio in the Stokes $I$ continuum ranges from 200 to almost 450 . In spite of the limited number of spectra obtained, we observed quite definite transient spectral features in the Stokes $I$ spectra of the first two sets. In Fig. 1, we show the differences between the individual Stokes $I$ spectra recorded during the second half-night (the best one of the three) and their average. A transient is clearly seen, with a complex structure moving through the line. The average Stokes $V$ spectra is uniformly featureless down to the noise limit, as are the differences between the individual Stokes $V$ spectra and this average.

In the following sections, we shall discuss the interpretation of those observations in terms of constraints on a possible magnetic field.

\section{Analytical interpretation}

When observing a transient spectral feature, the disk-integrated Stokes $I$ "intensity" observed at wavelength $\lambda$ in the line at time $t$ can be written as:

$$
\mathcal{F}_{I}(\lambda ; t)=\mathcal{F}_{I_{0}}(\lambda)+\Delta \mathcal{F}_{I}(\lambda ; t)
$$


$\mathcal{F}_{I_{0}}(\lambda)$ is the steady-state ("intrinsic") Stokes $I$ flux in the line:

$$
\mathcal{F}_{I_{0}}(\lambda)=\int_{-1}^{+1} d x \int_{-\sqrt{1-x^{2}}}^{\sqrt{1+x^{2}}} d y I\left(x, y ; \lambda-\lambda_{0}-\Delta \lambda_{R} x\right) .
$$

The formalism is similar to that employed by Mathys (1995). $x$ and $y$ are rectangular coordinates on the stellar surface, in a reference system whose origin is at the disk centre, and where the $y$ axis lies in the plane defined by the stellar rotation axis and the line of sight. $\Delta \lambda_{R}=(v \sin i / c) \lambda_{0}$, where $\lambda_{0}$ is the wavelength of the center of the line. $I$ is the steady-state intensity of the local emergent line at point $(x, y)$ of the stellar surface. We assume that the line profile transient is localized to a small region of the stellar surface centred around a point with coordinates $(\xi, \eta)$. Thus we can write:

$$
\begin{aligned}
\Delta \mathcal{F}_{I}(\lambda ; t) & =\int_{\xi-d \xi}^{\xi+d \xi} d x \int_{\eta-d \eta}^{\eta+d \eta} d y \Delta I\left(x, y ; \lambda-\lambda_{0}-\Delta \lambda_{R} x ; H(x, y) ; t\right) \\
& \approx \int_{\xi-d \xi}^{\xi+d \xi} d x \int_{\eta-d \eta}^{\eta+d \eta} d y \Delta I\left(\xi, \eta ; \lambda-\lambda_{0}-\Delta \lambda_{R} \xi ; H(x, y) ; t\right),
\end{aligned}
$$

where $H(x, y)$ is the intensity of the magnetic field at point $(x, y)$. The quantity $\Delta I$ is the difference between the instantaneous (at time $t$ ) and steady-state "intensities" at point $(x, y)$ at the same wavelength and at the same location on the stellar surface. The other notations are self-explanatory.

We assume that the Stokes $V$ flux in the line is zero outside the transient, so that it can be written:

$$
\mathcal{F}_{V}(\lambda ; t)=\Delta \mathcal{F}_{V}(\lambda ; t)
$$

with for $\Delta \mathcal{F}_{V}$ an expression similar to Eq. (3), with $I$ replaced by $V$. The quantity $\Delta V$ appearing in that expression is related to the quantity $\Delta I$ through:

$$
\begin{aligned}
& \Delta V\left(\xi, \eta ; \lambda-\lambda_{0}-\Delta \lambda_{R} \xi ; H(x, y) ; t\right) \\
& \quad \approx-\bar{g} \Delta \lambda_{Z} H_{z}(x, y) d \Delta I\left(\xi, \eta ; \lambda-\lambda_{0}-\Delta \lambda_{R} \xi ; t\right) / d \lambda,
\end{aligned}
$$

where $\bar{g}$ is the effective Lande factor of the transition, $H_{z}$ is the component along the line of sight of the magnetic vector at a point $(x, y)$, and $\Delta \lambda_{Z}=k \lambda_{0}^{2}$, with $k=4.67 \times 10^{-13} \AA^{-1} \mathrm{G}^{-1}$. Relation (5) is obtained under the weak field approximation (for details of its derivation, see Mathys 1989). This approximation implies that the separation of the individual magnetic line components is small with respect to the non-magnetic width of the emergent line. For a line such as He I $\lambda 6678$, which is rather broad in the absence of a magnetic field (due in particular to Stark broadening), the field can be considered as weak provided that it is not much stronger than $10 \mathrm{kG}$ : this does not introduce any practical restriction.

In the weak field approximation, $\Delta I$ is independent of the local field strength $H(x, y)$, so that Eq. (3) can be rewritten under the form:

$$
\Delta \mathcal{F}_{I}(\lambda ; t)=\alpha \Delta I\left(\xi, \eta ; \lambda-\lambda_{0}-\Delta \lambda_{R} \xi ; t\right),
$$

where $\alpha$ is the fraction of the projected stellar surface covered by the transient. Combining this relation with the expression of $\Delta \mathcal{F}_{V}$ and Eq. (5), we finally get:

$$
\Delta \mathcal{F}_{V}(\lambda ; t)=-\bar{g} \Delta \lambda_{Z} \frac{d \Delta \mathcal{F}_{I}(\lambda ; t)}{d \lambda}\left\langle H_{z}\right\rangle
$$


with

$$
\left\langle H_{z}\right\rangle=\frac{1}{\alpha} \int_{\xi-d \xi}^{\xi+d \xi} d x \int_{\eta-d \eta}^{\eta+d \eta} d y H_{z}(x, y) .
$$

$\left\langle H_{z}\right\rangle$ is the average over the region where the transient is localized of the component of the magnetic vector along the line of sight. Equation (8) shows that for a given value of this average, the observed circular polarization signal is maximum at wavelengths where the gradient of the Stokes $I$ transient is largest. These wavelengths can be easily identified on Fig. 1: we have derived upper limits of $\left\langle H_{z}\right\rangle$ in the transients from the comparison of the noise in the Stokes $V$ spectrum with the derivative of the Stokes $I$ transient profile on both flanks of the latter. From consideration of those observations with the steepest Stokes $I$ transients and the lowest noise Stokes $V$ spectra, we determine for $\left\langle H_{z}\right\rangle$ a $1 \sigma$ upper limit of $6 \mathrm{kG}$. We emphasize that because in this approach, we focus our attention on local, rather than global, magnetic fields, the signal that is useful to derive this upper limit is only a small fraction of the total intensity received from the star: this accounts to a large extent for the rather high limiting value.

\section{Numerical simulation}

To complement the analytical interpretation just presented, we have carried out some simple numerical simulations, which we report below.

Synthetic Stokes $V$ profiles of the line He I $\lambda 6678$ have been computed numerically for a number of configurations within the general framework of the following stellar model. The physical parameters in the atmosphere are given values representative of $\lambda$ Eri, and $v \sin i$ is taken equal to $310 \mathrm{~km} \mathrm{~s}^{-1}$. The star is assumed to be seen equator-on. There are two square "magnetic spots" of identical (but adjustable) area on its equator. The magnetic field in these two spots has a single strength and is radial, but each spot's field orientation may be directed either inward or outward. Effects of foreshortening and limb darkening are included. The emergent Stokes $V$ line profile is computed locally at a number of grid points within a spot by solving the scalar transfer equation in the usual way (that is, neglecting the magnetic field), then shifting the result to the central wavelength of each $\sigma$ component and scaling the intensity according to the relative strength of that component, and finally subtracting the $\sigma_{-}$from $\sigma_{+}$. In other words, a numerical solution of the (vectorial) transfer equation for polarized light is not computed; instead the approach corresponds to adopting a weak line approximation solution of that equation. The resulting local Stokes $V$ profiles are then numerically integrated over a grid of points covering the stellar disk (Stokes $V$ is assumed to be uniformly 0 outside the two spots).

We give hereafter a few examples of the results obtained with this approach. Figure 2 shows how the detectability of the magnetic field depends on the position of the spots on the surface. It features Stokes $V$ line profiles computed for two magnetic spots with a longitude extension of $1 \%$ of the stellar disk diameter, and with centres separated from each other by a distance $\Delta \xi=0.32$ (units are stellar radii projected on the plane perpendicular to the line of sight). The magnetic field strength in both spots is $10 \mathrm{kG}$; in one of them, the field points outwards; in the other it is directed inwards. Three curves are shown, corresponding respectively to a location of the mid-point between the two spots 


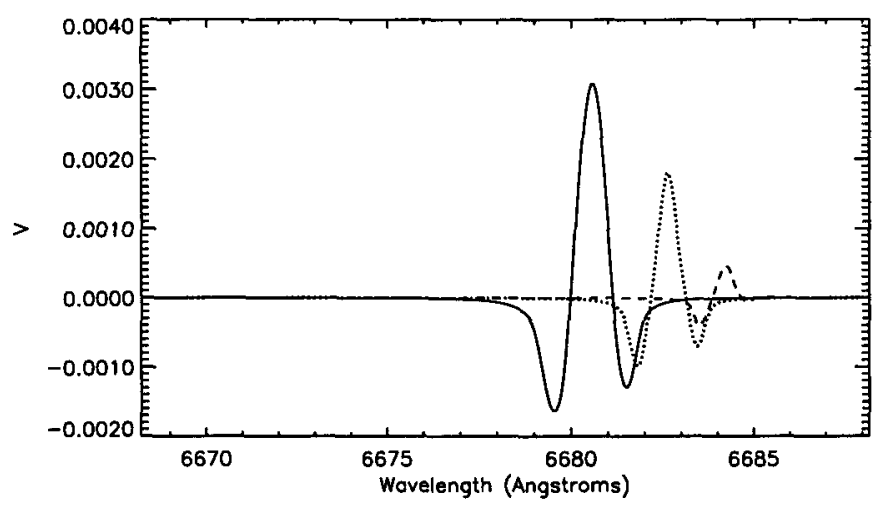

Figure 2. Dependence of Stokes $V$ signal on the longitude of the magnetic spots (see text for details).

at longitudes $20^{\circ}$ (solid line), $40^{\circ}$ (dots), and $60^{\circ}$ (dashes) on the receding part of the stellar hemisphere (the plane defined by the line of sight and the stellar rotation axis being taken as the longitude origin). One can clearly see how the polarimetric signal decreases as the spots travel away from disk centre.

In the last example, the separation of the spots was taken so as to bring the positive part of the $V$ profiles for each spot to the same wavelength, so their contributions add up and make the detection easier. This effect is illustrated in Fig. 3, where we show the profiles generated by two spots symmetrically located on each side of disk centre, each extending over $3 \%$ of the stellar diameter, and in which magnetic fields of the same strength $(10 \mathrm{kG})$ but of opposite polarities are present, for two spot center separations, $\Delta \xi=0.32$ and 1.0. The detection is more difficult for larger separations, for which the positive parts of the Stokes profiles do not align in wavelength. We also note that similar cancellation effects mean that the detection threshold value decreases more slowly for large spots than the gain afforded by a larger area. This fact means that, per unit area, it is easier to detect small spots per unit area. The cancellation effect is also the key reason ultimately why the Zeeman effect becomes difficult to observe in a rapidly rotating star with a radial magnetic field.

For direct comparison with the observations, we added random noise to the synthetic profiles. Figure 4a shows the numerical result obtained with a noise of 0.0018 (in units of the Stokes $I$ continuum level), for two spots at disk centre with a longitude extension of 0.03 disk diameter, $3 \mathrm{kG}$ magnetic fields of opposite polarities, and a separation of 0.16 stellar radius. It is compared in Fig. $4 \mathrm{~b}$ with one of our best observations (what is represented is the difference between an individual Stokes $V$ recording and the average of all the recordings of the corresponding night). The similarity between the two plots is noteworthy. It indicates that the kind of magnetic field considered in the model could be present in the star and be near the threshold of detection.

This example is statistically significant to a few sigma, but continuum placement problems in reality would degrade this considerably. If as observers we detected this particular signature in the corresponding intensity spectrum, and 


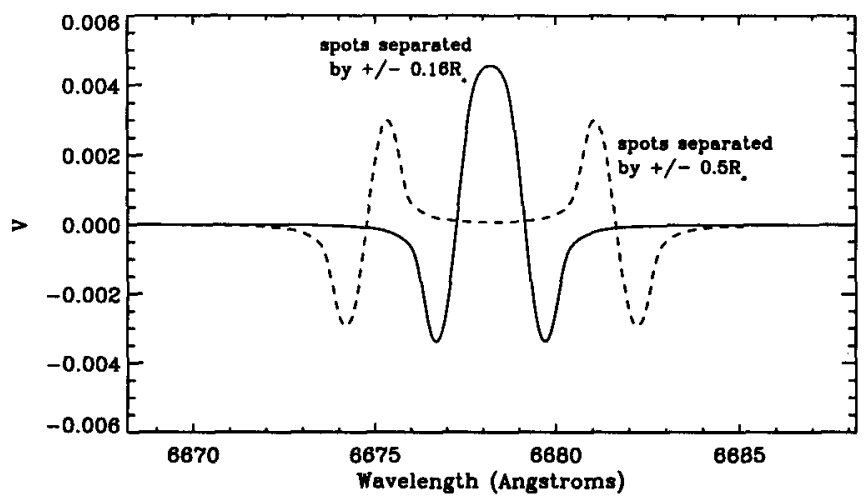

Figure 3. Dependence of Stokes $V$ signal on the separation of the magnetic spots (see text for details).
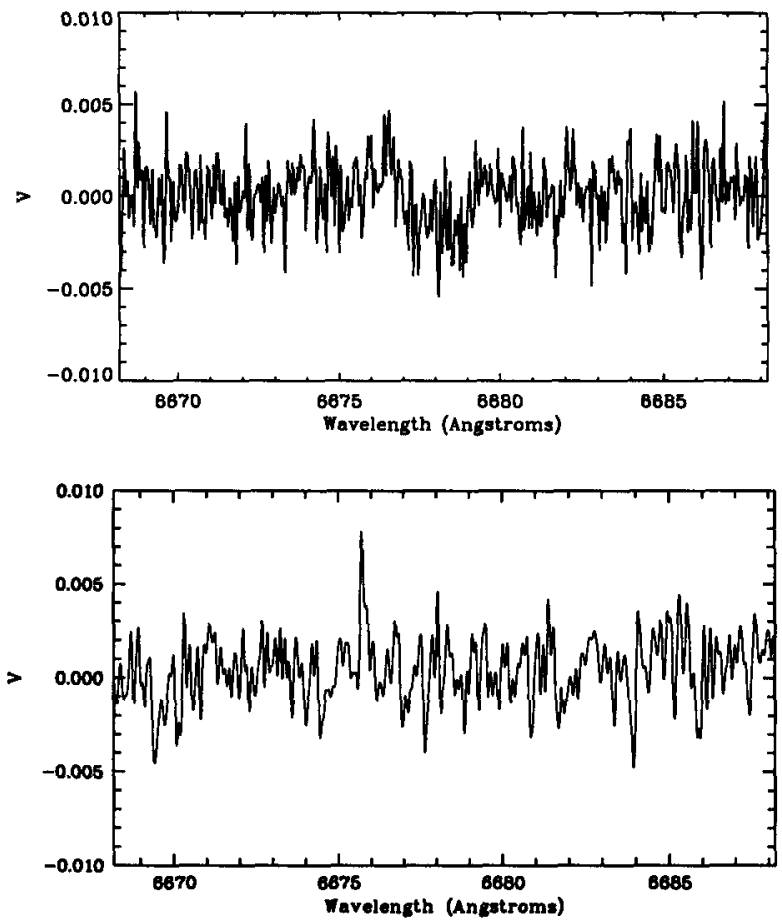

Figure 4. Top: (a) synthetic spectrum with noise for two spots around disk center with opposite fields of strength $3 \mathrm{kG}$, extending over 0.03 diameter of the stellar disk and separated by 0.16 radius. Bottom: (b) actual observations: difference between one Stokes $V$ spectrum and the average of all the Stokes $V$ spectra taken during the same night. 
at the same wavelength where a line profile transient occurred, we would consider it just detectable. Thus, it is in reasonable agreement with our analytical analysis. In reality one would insist upon several examples before making any such formal first claim of Zeeman signature in a rapidly rotating Be star.

\section{Conclusion}

The original approach described in this note to detect localized, transient magnetic fields in $\lambda$ Eri, associated with transient spectral features in the line $\mathrm{He} I$ $\lambda 6678$, rules out the presence of such fields with strengths in excess of $6 \mathrm{kG}$ and covering effective areas of a few percent. The detection limit does, as a matter of fact, depend critically on the structure of the postulated magnetic regions, but the present study is definitely consistent with the possible existence of fields of $3 \mathrm{kG}$ or less, which should be near the detection limit if they occur in pairs of oppositely magnetized spots arranged in at favorable longitudinal intervals on the star's disk. This level of field strength is fully compatible with the estimates that have been made on the basis of theoretical considerations aimed at providing a magnetic interpretation of a number of variable phenomena observed in $\lambda$ Eri. In other words, the observational results reported here do not provide a critical test of these interpretations. This study has mostly reached the limits of what is currently feasible in the considered direction of investigation, in particular by relying on what are state-of-the-art observations. Therefore, the potential of the applied approach for further progress in constraining the magnetic fields of typical (i.e., fast rotating) Be stars appears limited. This motivates us to consider as a next step potential techniques which rely diagnosis on other processes than the Zeeman effect for magnetic field, such as the space-based Hanlé technique recently developed by Ignace, Nordsieck, \& Cassinelli (1997). Such so-far novel approaches seem to hold the best promises for the future of magnetic field studies in Be stars, and in hot stars with strong winds.

\section{References}

Babcock, H.W., 1958, ApJS, 3, 141

Barker, P.K., Landstreet, J.D., Marlborough, J.M., Thompson, I.B., 1985, ApJ, 288,741

Ignace, R., Nordsieck, K.H., Cassinelli, J.P., 1997, ApJ, 486, 550

Mathys, G., 1989, Fundam. Cosmic Phys., 13, 143

Mathys, G., 1995, A\&A, 293, 733

Mathys, G., Hubrig, S., 1997, A\&AS, 124, 475

Smith, M.A., Polidan, R.S., 1993, ApJ, 408, 323

Smith, M.A., Grady, C.A., Peters, G.J., Feigelson, E.D., 1993, ApJ, 409, L49

Smith, M.A., Hubeny, I., Lanz, T., Meylan, T., 1994, ApJ, 432, 392

Smith, M.A., Plett, K., Johns-Krull, C.M., Basri, G.S., Thomson, J.R., Aufdenberg, J.P., 1996, ApJ, 469, 336 